gestalterisches Potential erfahren, spüren sie ihre Wirkmächtigkeit. In Kunst und Kultur werden andere Lebensentwürfe sichtbar, ist Wandel erfahrbar, prüfbar und durchspielbar, eröffnen sich Perspektiven, entsteht Neues.

Wissen teilen. Wissenschaftliche Bibliotheken sind der Platz, an dem eine selbstständige, kritische Auseinandersetzung mit dem kulturellen, wissenschaftlichen und künstlerischen Denken und Agieren in großer Breite und historischer Tiefe möglich ist. Wissenschaftliche Aussagen und Diskussionen können hier anhand entsprechender Publikationen geprüft und nachvollzogen werden. Die Vielfalt der methodischen Ansätze, die diskursive Herausbildung der Erkenntnisse und die Grenzen der gegebenen Erklärungen werden in den Publikationen fassbar. Und natürlich ist wissenschaftliches Arbeiten ohne die Berücksichtigung der einschlägigen Publikationen unmöglich.
Kontexte herstellen. Durch die Fülle ihres Materials gelingt es in der Bibliothek, die Bedingungen unserer Gesellschaften, Alltagskulturen, aber eben auch wissenschaftliche, künstlerische, politische Auseinandersetzungen verstehbar zu machen. Die Wahrnehmung dieser Bezüge ist die Grundlage, wenn man nicht in teilmythologische Erklärungsgebilde zurückfallen oder verheerende Simplifizierungen attraktiver erscheinen lassen möchte.

Sicher können Sie noch weitere Gesichtspunkte beisteuern, haben Sie besondere Beispiele. Ich würde mich freuen. Wir werden in den nächsten Monaten verstärkt den Dialog suchen, um den Resonanzraum der Kultur für die politischen Entscheidungsträger besser wahrnehmbar zu machen. Wir brauchen Ihr Mittun! Teilen Sie Ihre Erfahrungen und Überzeugungen, sprechen Sie mich an oder schicken Sie mir Ihre Beispiele, Ideen oder Unterstützungsmöglichkeiten!

Rupert Schaab

\title{
Hölderlin 2020 in der WLB, Teil 2
}

\section{Ausstellung „Aufbrüche - Abbrüche. 250 Jahre Friedrich Hölderlin"}

Der Ausstellungstitel „Aufbrüche - Abbrüche“ wurde gewählt, um zentrale, sich wiederholende Merkmale von Hölderlins Leben, Werk und Rezeption widerzuspiegeln, woran sich die Themen der Ausstellung anknüpfen, die bereits im letzten Heft des WLBforum beschrieben wurden. Wie sich herausstellte, wurde der Titel aber ebenso zum Sinnbild der gesamten Zeit, in der die Jubiläumsausstellung die Arbeit der Bibliothek und speziell des Hölderlin-Archivs begleitet hat - von den komplexen Vorbereitungen der Ausstellung über die sich immer wieder verändernden Rahmenbedingungen bis hin zur Corona-Pandemie. Der hoffnungsvolle Aufbruch in das große Jubiläumsjahr 2020 mündete schließlich in einen radikalen Abbruch der Aktivitäten, bedingt durch die in dieser Form nicht für möglich gehaltene Pandemieentwicklung im Frühjahr 2020 und den plötzlichen Lockdown.

Doch dieser Stillstand währte nur kurz. Es deutete sich schon schnell wieder ein neuer Aufbruch an. Durch die notwendige Verschiebung der Ausstellung eröffnete sich auch die Chance auf eine Präsentation der Ausstellung im neuen Erweiterungsbau, wie es bereits fester Bestandteil der ersten Planungen war. Verbunden damit waren natürlich wieder erneute Umgestaltungen, um die Ausstellung den neuen räumlichen Bedingungen anzupassen. Die Arbeit ging also während der Corona-Krise kontinuierlich weiter. Und durch wiederholte Verzögerungen bei der Inbetriebnahme des Erweiterungsbaus wurden auch die Planungen des Aufbaus im Sommer erschwert, sodass sich die an der Vorbereitung Beteiligten bis zur Eröffnung der Ausstellung stets neuen Herausforderungen gegenübergestellt sahen.

Ganz besonders zu danken ist deshalb den Mitarbeiterinnen des Hölderlin-Archivs, insbesondere Ulrike Seegräber und Angelika Votteler für ihren unermüdlichen Einsatz, ferner der Ausstellungsreferentin, Dr. Ida Bentele, die bis zu ihrem Abschied im Juli 2020 
die Ausstellung mit gesteuert hat, des Weiteren der Restauratorin Sonja Brandt für die konservatorische Betreuung und schließlich Ina Bauer, die - in Zusammenarbeit mit Simon Busse - für die grafische Gestaltung der Ausstellung verantwortlich war und für die wechselhaften Rahmenbedingungen immer wieder neue Lösungen fand. Ein großer Dank gilt dem Ministerium für Wissenschaft, Forschung und Kunst Baden-Württemberg sowie der BW-Stiftung für die finanzielle Unterstützung, welche die Ausstellung überhaupt erst möglich machte. Im Herbst scheint diese "Odyssee” endlich an ihr Ziel zu gelangen. So soll am 12. Oktober die Ausstellung feierlich eröffnet werden, freilich wegen der Corona-Auflagen leider mit deutlich weniger Gästen als ursprünglich vorgesehen. Ministerin Theresia Bauer wird das Grußwort sprechen, Professor Thomas Knubben mit dem Festvortrag "Hölderlin 21 " auf die Ausstellung einstimmen und Nikola Diskič (Bariton) sowie Alexander Fleischer (Flügel), organisiert von der Internationalen Hugo-Wolf-Akademie, für die musikalische Umrahmung sorgen. Leider kann durch diese Verschiebung die Ausstellung nicht mehr wie geplant im Anschluss in der Bibliothèque nationale et universitaire in Straßburg präsentiert werden, da dort nur im Herbst entsprechende Zeiträume frei waren. Zur besseren Vermittlung der Themen der Ausstellung für die französischsprachige Zielgruppe wurden jedoch die Fachbeiträge des Katalogs ins Französische übersetzt und als Ergänzung diesem beigelegt.

Der Großteil der Begleitveranstaltungen konnte in den Herbst verlegt werden. Den Auftakt machen Karl Heinz Ott und Prof. Dr. Manfred Koch am 19. Oktober, die in einer Podiumsdiskussion Karl Heinz Otts viel beachtetes Buch „Hölderlins Geister" beleuchten. Es folgt im wöchentlichen Rhythmus ein abwechslungsreiches Programm:

\section{Oktober 2020, $19 \mathrm{Uhr}$}

\section{Vortragssaal der Württembergischen Landesbibliothek \\ Karl-Heinz Ott: Hölderlins Geister \\ Mit dem Autor Karl-Heinz Ott und Prof. Dr. Manfred Koch}

Zum Hölderlin-Jubiläum hat Karl-Heinz Ott ein feinsinniges, essayhaftes Buch über den Dichter geschrieben. ıHölderlins Geister haben viele Facetten. Neben werkbezogenen Aspekten wie seine dichterische Sprache und sein Griechenlandbild sowie biografischen Gesichtspunkten wie seine Krankheit wird insbesondere die Vielseitigkeit der Rezeption Hölderlins im 20. Jahrhundert und seine Bedeutung für die Moderne thematisiert. Über diese Facetten diskutiert der Autor Karl-Heinz Ott in einem kontroversen Gespräch mit dem Literaturwissenschaftler Manfred Koch.

\section{Oktober 2020, $19 \mathrm{Uhr}$}

Vortragssaal der Württembergischen

Landesbibliothek

HÖLDERLIN. Bilder-Klänge-VerDichtung: Intermediale Performance mit Werner Englert (Musik), Katharina Hoehler (Malerei) und

\section{Dieter E. Neuhaus (Schauspiel/Dramaturgie)}

Diese Begegnung dreier Künstler mit dem Dichter Hölderlin und seinem Werk verspricht, die Faszination Hölderlin in einer intermedialen Live-Performance dem Publikum zu vermitteln. Verse und Gedanken Friedrich Hölderlins werden zu Bildern, zu musikalischen Klängen eines Instruments, einer Stimme, lassen Interaktionen zwischen den Künsten entstehen. Die Auseinandersetzung mit einem Menschen und seiner Dichtung aus einer vergangenen Epoche geschieht in einem Augenblick und schafft einen besonderen, ungewöhnlichen Zugang. Befremdliches wird neu entdeckt, zugleich aber auch Vertrautes freigelegt.

Die intermediale Performance ist Teil des Literatursommers, der sich 2020 Hölderlin und Hegel widmet. Die Veranstaltung kann mit Sondermitteln aus diesem Landesprojekt finanziert werden.

\section{November 2020, $19 \mathrm{Uhr}$}

Vortragssaal der Württembergischen Landesbibliothek

Vertonungen von Friedrich Hölderlin

Ein Liederabend mit Peter Schöne (Bariton) und Alexander Fleischer (Klavier) in Kooperation mit der Hugo-Wolf-Akademie

Als leuchtender Bariton von der Süddeutschen Zeitung gepriesen, als exzellenter Sänger mit hervorragender Technik von FAZ, Opera Now und Opernwelt geadelt, hat sich Peter Schöne mit seinem ausdrucksvollen lyrischen Bariton als Opern-, Konzert- und Liedsänger national und international etabliert. Alexander Fleischer ist Preisträger des Internationalen Wettbewerbs für Liedkunst, den die Internationale Hugo-Wolf-Akademie alle zwei Jahre in Stuttgart veranstaltet. 
12. November 2020, $18 \mathrm{Uhr}$

Vortragssaal der Württembergischen Landesbibliothek

Rezitationsabend in Kooperation mit der Akademie für gesprochenes Wort

"So komm! daß wir das Offene schauen,

Daß ein Eigenes wir suchen, so weit es auch ist."

Eine Einladung, Hölderlins gesprochene Sprache zu erleben.

Die unmittelbarste Annäherung an Hölderlin besteht darin, inn selbst zur Sprache kommen zu lassen, seine Sprache aufzunehmen und in das Freie, Weite und Offene aufzubrechen. In dieser Veranstaltung werden vor allem Texte aus seiner Stuttgarter Zeit im Blickpunkt stehen.

\section{November 2020, $18 \mathrm{Uhr}$}

Vortragssaal der Württembergischen Landesbibliothek

Hans Gerhard Steimer: Friedrich Hölderlins Homburger Folioheft in diachroner Darstellung Mit Handschriften von Schriftstellern befassten sich traditionell vor allem Editoren. Ihr Interesse galt dem Text. Das Dokument als solches stand allenfalls bei Autografensammlern oder Archivaren im Fokus. Das änderte sich mit dem Aufkommen Faksimile-basierter Editionen. Die digitalen Medien bieten wiederum neue Möglichkeiten der Visualisierung von Manuskripten. Ein innovatives Beispiel ist die diachrone Darstellung des Homburger Foliohefts, der wichtigsten Sammelhandschrift im Spätwerk Hölderlins.

\section{November 2020, $19 \mathrm{Uhr}$}

Vortragssaal der Württembergischen Landesbibliothek

Hölderlin-Vertonungen von Luigi Nono und Hans Zender

\section{Ein Konzert mit dem Kairos Quartett in} Kooperation mit der Hugo-Wolf-Akademie

Das in Berlin ansässige Kairos Quartett widmet sich seit seiner Gründung 1996 vornehmlich der Musik des ausgehenden 20. und des 21. Jahrhunderts und ist damit das erste derartig ausgerichtete Streichquartett auf dem europäischen Festland. Zu seinem Repertoire gehören auch Kompositionen von Luigi Nono und Hans Zender zu Vertonungen Friedrich Hölderlins, die es an diesem Konzertabend anlässlich des Hölderlin-Gedenkjahres vorstellt.

\section{Dezember 2020, $19 \mathrm{Uhr}$}

Vortragssaal der Württembergischen

Landesbibliothek

FRIEDRICH HÖLDERLIN / DICHTER SEIN. UNBEDINGT!

Buch und Regie: Hedwig Schmutte und Rolf Lambert in Ko-Produktion mit SWR und Arte

Der Film rekonstruiert das dramatische Leben des Friedrich Hölderlin. Vor dem Hintergrund elementarer historischer Umwälzungen und entlang der zentralen Lebensstationen erzählt er die Geschichte einer künstlerischen Radikalisierung.

\section{Dezember 2020, 19 Uhr}

Vortragssaal der Musikhochschule "O Asia, das Echo von dir" - Hölderlin und

Beethoven in Wort, Szene und Musik Mit Corinna Harfouch (Rezitation) und Hideyo Harada (Klavier)

Kann es Zufall sein, dass Beethoven und Hölderlin das Geburtsjahr teilen? Sie haben Werke von eruptiver Kraft geschaffen, Grenzen überwunden und Konventionen gesprengt. Hölderlins ekstatische Lyrik und Beethovens subjektive Klangsprache waren in der Lage, die Zeitgenossen zu verstören. Verblüffend gleichtönend sind auch die Schlagworte für beide Biografien. Isolation lautet eines davon, Revolution ein weiteres: Für die Französische Revolution können sie sich begeistern - und sind gleichermaßen tief enttäuscht von deren Ausgang. Es folgt für beide die Suche nach anderen Strömungen, in denen sie ihre humanistischen Ideale verwirklicht finden können. Beider Sehnsuchtsort ist der Orient. Im 21. Jahrhundert ist ihr gemeinsamer gedanklicher Horizont aktueller denn je - ihre Auseinandersetzung mit geistigen Konzepten des Orients, der sich abzeichnende Dialog der Kulturen. Eine Textcollage aus Briefen und Tagebuchaufzeichnungen Beethovens, Gedichten und Selbstzeugnissen Hölderlins sowie Auszügen aus dessen Roman ıHyperions treffen auf musikalische Werke aus der mittleren und späten Schaffensperiode des Wiener Klassikers.

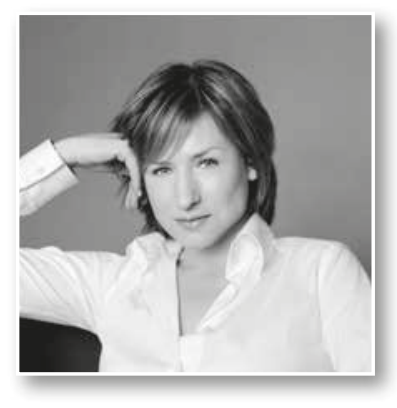

Corinna Harfouch; Foto: CDirk Dunkelberg

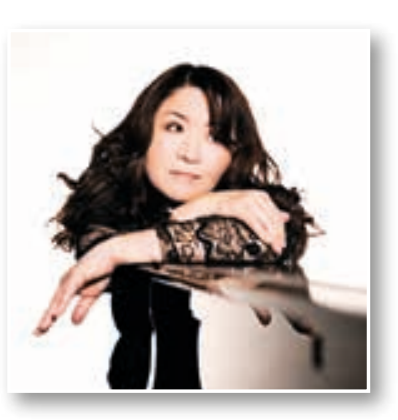

Hideyo Harada; Foto: @Uwe Arens 
9. Dezember 2020, $19 \mathrm{Uhr}$

\section{Vortragssaal der Württembergischen}

Landesbibliothek

Der Not ist jede Lust entsprossen -

ein Konzertabend auf Spuren Hölderlins mit

Susanne Stock (Akkordeon), Anne Schneider

(Gesang), Urs Stämpfli (Schauspiel),

Astrid Alexander (Audio)

Genie. Zweifler. Wanderer. Vier Künstler*innen nähern sich Friedrich Hölderlin in einem performativen literarischen Konzertprojekt. Mit Hölderlins Texten, Neukompositionen und vorhandenen, adaptierten Vertonungen, mit eigenen Reflexionen und szenischen Aktionen - verbunden durch eingespielte Fieldrecordings - treten sie in einen zeitgenössischen Diskurs mit dem jungen Dichter und seiner Kunst.

\section{Kreuzworträtsel zum Hölderlin-Jahr}

Wir haben uns über die rege Beteiligung am Kreuzworträtsel anlässlich des Hölderlin-Jahres gefreut. Hier sind die Lösungsworte: HEGEL SCHELLING. Mit den beiden berühmten Philosophen hat Hölderlin im Tübinger Stift zusammen studiert und vom Herbst 1790 bis Sommer 1793 ein Zimmer geteilt.
Die glücklichen Gewinnerinnen und Gewinner erhalten einen Katalog zur Jubiläumsausstellung.

Jörg Ennen

\section{Aiga Rasch - Stuttgarter Künstlerin und Illustratorin}

Am 9. Juli 1941 erblickt Aiga Rasch das Licht der Welt und wird in eine kreative Familie hineingeboren. Ihr Großvater Karl Alfons Nägele war Maler, ihre Mutter Lilo Rasch-Nägele Modegrafikerin und Malerin und ihr Vater Bodo Rasch ebenso wie ihr Onkel Heinz Rasch als erfolgreicher Architekt tätig. Während des Zweiten Weltkriegs mangelt es in Aigas Kindheit an Spielzeug und schon früh dienen inr die ausrangierten Farbpaletten und gebrauchte Pinsel der Mutter als alltäglicher Spielersatz.

Von 1953 bis 1961 besucht sie das Fanny-LeichtGymnasium in Stuttgart und das Fach Kunst stellt mit der Gesamtleistung "gut" ihre beste Note im Reifezeugnis dar. Bereits in der Schulzeit gelingt es ihr, illustrierte Kurzgeschichten in lokalen Zeitungen zu veröffentlichen. Sie interessiert sich früh für Comics und ist stolz auf ihre Sammlung, in der sich u.a. Superman, Tarzan und Prinz Eisenherz tummeln.

Im Jahr 1961 fängt sie an der Universität Tübingen an zu studieren, bricht ihr Philosophie-Studium aber nach nur einem Semester ab. Ein Jahr später besucht sie den Vorbereitungskurs der Staatlichen Akademie der Künste in Stuttgart, jedoch wird ihr vom Beginn des Kunststudiums durch den betreuenden Professor Hugo Peters abgeraten. Zwar bescheinigt er ihr, sie besitze Talent, doch sei ihr feministischer Stil zu sehr von der erfolgreichen Mutter geprägt und sie solle sich stattdessen autodidaktisch weiterentwickeln. Ihr grafisches Handwerkszeug schärft sie daraufhin in ihrer achtzehnmonatigen Arbeit für das neu gegründete Verbrauchermagazin DM unter dem Chefgrafiker Hans Pape.

Aiga Rasch erhält 1963 ihren ersten Buchauftrag als freischaffende Illustratorin für den Kalender "heute morgen übermorgen 1964 - Taschenkalender für junge Mädchen" beim Franckh-Kosmos Verlag: Sie kreiert das Cover und die Innenillustrationen. Im gleichen Jahr folgen zwei weitere Aufträge und die junge Grafikerin etabliert sich bei jenem Stuttgarter Verlagshaus in den nächsten Jahren als feste Größe. 